Structural Eng. / Earthquake Eng. Vol.6. No.2, 395s-404s. October 1989

Japan Society of Civil Engineers (Proc. of JSCE No.410/I-12)

\title{
ON THE QUANTITATIVE RELATION BETWEEN EFFECTIVE FOURIER SPECTRUM OF INPUT EARTHQUAKE MOTIONS AND ENERGY RESPONSE SPECTRUM OF SDOF STRUCTURES
}

\author{
By Kiyoshi HIRAO*, Yoshifumi NARIYUKI**, Shuji SASADA*** and Junichi MASUI****
}

\begin{abstract}
The purpose of this study is to investigate a method of quantitative estimate of the elastic input energy $E_{i e}$ and hysteretic energy $E_{h p}$ of SDOF structures subjected to strong earthquake motions. In this study, numerical response analyses for bi-linear SDOF structures were carried out by the use of artificial earthquakes and recorded ones. From the examination of analytical results, it is found that a good correlation can be seen between the energy response spectrum $E_{s}$ for $E_{i e}$ and $E_{h \rho}$ and effective Fourier amplitude spectrum $F_{s e}$ for each input earthquake. Also the difference in values for the ratio of the square root of the $E_{s k}$ to $F_{s e k}$, at any natural period $T_{k}$, is seen to be substantially reduced amongst the earthquakes. Considering these results, the authors derived regression equations for the mean value of this ratio, $\sqrt{E_{s}} / F_{s e}$, for the artificial earthquakes relating to structural parameters, i.e., natural period $T$, damping factor $h$, yield strength ratio $R$ and secondary slope $p$.

Keywords: seismic damage, energy response, SDOF structures
\end{abstract}

\section{INTRODUCTION}

Recently, input energy and hysteretic/plastic strain energy of the structures subjected to strong earthquake motions have attracted special interest because they are amongst the best parameters to evaluate the damage on structures, and many studies on these energies have been carried out ${ }^{1) \sim 11)}$. From these studies, the qualitative and quantitative relation between these input and hysteretic energies and structural parameters (such as natural period, damping factor, yield strength and characteristics of restoring force) was made quite clear $^{2)}$ (7). Also the relationship between these energies and the characteristics of input earthquake motion, i. e., the frequency content, duration, total power, etc., was examined, and the following matters concerning this relationship were pointed out ; 1) for the ratio of the hysteretic energy $E_{h \rho}$ to elastic/inelastic input energy $E_{i}\left(E_{h \rho} / E_{t}\right)$, the effects of the input earthquake motion on both energies cancel each other out and scattering of this ratio due to the difference of the earthquakes decreases ${ }^{3), 77}$. 2) The value of the input energy and the cumulative plastic deformation increase as the duration of the input earthquake motion lengthens ${ }^{9}$, and the cumulative plastic deformation takes on similar value irrespective of the difference of the input earthquake motions when the total power of each earthquake is the $s \mathrm{e}^{9}$. 3) In the case that the input earthquake motions are classified into groups by means of their maximum acceleration $A$ to maximum velocity $V$ ratio $(A / V)$, the value of the hysteretic energy for the group bearing the small $A / V$ becomes larger than that for the group with the large $A / V^{10)}$.

* Member of JSCE, Dr. Eng., Professor, Dept. of Civil Eng. , University of Tokushima (Minamijosanjima 2-1, Tokushima, 770, Japan)

** Member of JSCE, M. Eng., Lecturer, Dept. of Civil Eng., University of Tokushima (ditto)

*** Member of JSCE, M. Eng., Assistant, Dept. of Civil Eng., Anan College of Technology (Aoki 265, Anan, 774, Japan)

**** Member of JSCE, M. Eng., Nippon Telegraph and Telephone Corporation Ltd., Formerly Graduate Student, Dept. of Civil Eng., University of Tokushima 
4) A good correlation can be seen between the periodical characteristics for the energy response spectrum of $E_{i}$ and $E_{h \rho}$ and those in the Fourier spectrum for each corresponding input earthquake motion ${ }^{11}$. 5) In the case of earthquakes with similar Fourier spectrum, there is a near proportional relation between the value of the elastic input energy $E_{i e}$ and hysteretic energy $E_{h p}$ and total power $P_{t}$ of the input earthquake motion $^{11)}$. From these results, the qualitative effect of the characteristics of the input earthquake motions on elastic/inelastic input energy and hysteretic energy of the structures is gradually being brought out. However, these studies alone are not enough to develop an actual aseismic design procedure based on the energy concept, and there are two problems to be solved. One is how to develop a practical way to estimate the energy absorbing capacity of a structure and the other is how to quantitatively estimate the effect of the difference in characteristics of the input earthquake motions on the input energy and hysteretic energy of the structures with different structural parameters.

As this is a fundamental study relating to the latter problem, energy response analysis for wide ranging bi-linear SDOF structures was carried out by the use of twenty artificial earthquake motions and five recorded ones. These vary in frequency characteristics and duration. For each input earthquake, effective Fourier amplitude spectrum $F_{\text {se }}$ (described below) was also calculated. Then, referring to our previous study ${ }^{11}$, the authors examined the mutual relation between the effective Fourier spectra $F_{s e}$ for the input earthquakes and the response spectra $E_{s}$ for the elastic input energy $E_{i e}$ and hysteretic energy $E_{h p \text {. Lastly }}$ they derived regression equations for the mean value of the ratio $\sqrt{E_{i e}} / F_{s e}$ and $\sqrt{E_{h \rho}} / F_{s e}$ for all artificial earthquakes. These equations are available to estimate the value of $E_{i e}$ or $E_{h \rho}$ of the given structures comparatively accurately when the value of their structural parameters and effective Fourier spectrum $F_{s e}$ of the input earthquake motion are already known.

\section{INPUT EARTHQUAKE MOTION}

In order to maintain the generality of the analytical results, the artificial earthquakes Nos. 1 to 20 were

Table 1 Data of artificial earthquakes.

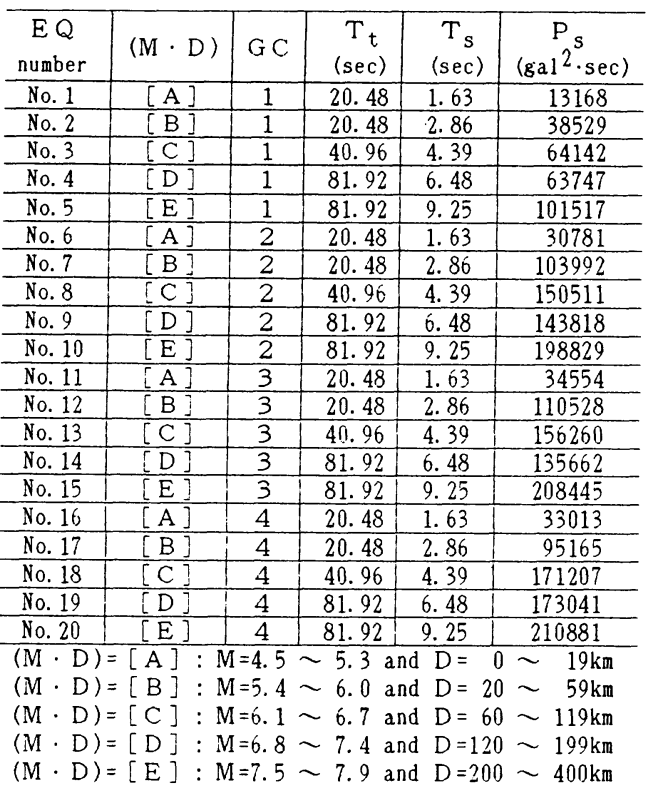

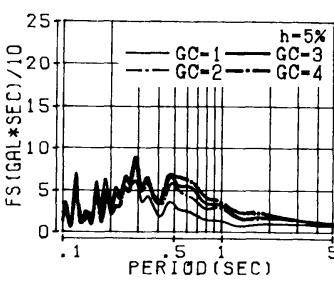

(a) $(M \cdot D)=A$

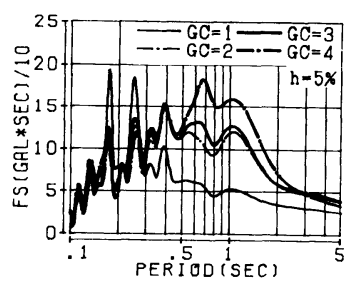

(c) $(M \cdot D)=C$

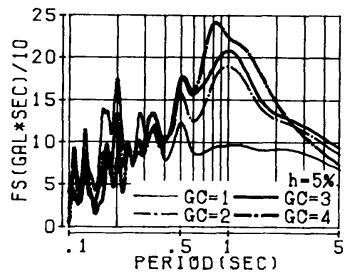

(e) $(M \cdot D)=E$

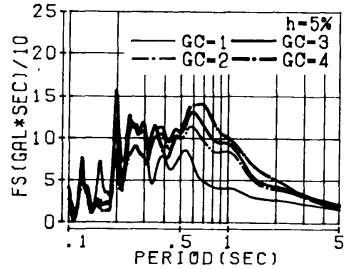

(b) $(M \cdot D)=B$

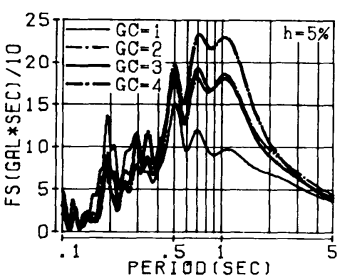

(d) $(M \cdot D)=D$

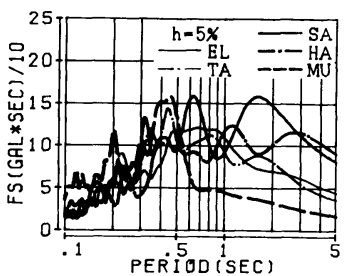

(f) Recorded
Fig. 1 Fourier amplitude spectra for input earthquake motions. 
generated $^{13) .14)}$ such that each of their acceleration response factor spectrum agrees with the target one shown in the Japanese specifications for road bridges $\mathrm{V}^{12}$. Table 1 shows the magnitude $M$, epicentral distance $D$ and ground condition GC (Group 1 to 4$)^{12)}$ for each spectrum targeted in each artificial earthquake. It also shows the total duration $T_{t}$, the duration of the strong motion $T_{s}$ and the power $P_{s}$ for the duration $T_{s}$. The symbols $[\mathrm{A}],[\mathrm{B}],[\mathrm{C}],[\mathrm{D}]$ and $[\mathrm{E}]$ in Table 1 find meaning within the scope of the value for the combination ${ }^{12)}$ of $M$ and $D$, respectively, as shown at the bottom of the table. The duration $T_{t}$ and $T_{s}$ was also determined by the method proposed in Ref. 14). Fourier spectra of these earthquakes, after having been smoothed by a Parzen window with band width $0.5 \mathrm{~Hz}$, are shown in Figs. 1 (a) to (e). From these figures and Table 1, it is noted that, corresponding to the large or small values of the targeted spectra and depending on the values of the magnitude and epicentral distance $(M \cdot D)$ and ground condition $\mathrm{GC}$, the value of the power $P_{s}$ and Fourier spectrum for long period range becomes larger with the increasing number of $\mathrm{GC}$ when $(M \cdot D)$ is the same as $(M \cdot D)=[\mathrm{A}],[\mathrm{B}],[\mathrm{C}],[\mathrm{D}]$ or $[\mathrm{E}]$. Also these values increase as the value of $(M \cdot D)$ gets larger when $\mathrm{GC}$ is the same as $\mathrm{GC}=1,2,3$ or 4 . From these facts, it is said that the earthquakes generated in this study differ from one another in frequency characteristics and power/duration. Moreover, in order to examine the corresponding relation between the analytical results for these artificial earthquakes and those for recorded ones, the next five recorded earthquakes were also used as the input earthquake motions; (a) Muroran S-241, N-S, Tokachioki Earthquake (1968), (b) Hachinohe S-252, N-S, Tokachioki Earthquake (1968), (c) El-Centro S 00 E, Imperial Valley Earthquake (1940), (d) Taft S 69 E, Kern Country Earthquake (1952) and (e) Los Angeles N00 W, San Fernand Earthquake (1971). As shown in Fig. 1 (f), the frequency characteristics for these five earthquakes are different from one another, as are the artificial earthquakes.

\section{STRUCTURAL PARAMETERS AND ANALYTICAL METHOD}

In this study, natural period $T$, damping factor $h$, yield strength ratio $R$ and secondary slope $p$ were adopted as the structural parameters of SDOF structures with bi-linear restoring force characteristics as shown in Fig. 2. Then, considering the ordinary values of actual structures, the following three values for the damping factor $h$ were used, 2.5, 5.0 and $7.5 \%$. For the natural period $T$, after dividing the range for $T=0.1$ to $5 \mathrm{sec}$. into nineteen equal parts on the logarithmic axis, twenty different values were employed. As for the secondary slope $p$ which is the ratio of plastic stiffness to elastic stiffness (see Fig. 2), the three values, $0.0,0.25$ and 0.5 , were selected. Three values of the yield strength ratio $R$ defined by equation ( 1$), 0.25,0.5$ and 0.75 , were also selected to correspond to each case where the inelastic response is strong, intermediate and weak, respectively.

$$
R=Q_{y} / Q_{e \max }=X_{y} / X_{e \max }
$$

in which $Q_{y}$ and $X_{y}$ are the yield strength and displacement, $Q_{e \max }$ and $X_{e \max }$ are the maximum restoring force and displacement obtained from the elastic response analysis, respectively.

The analytical method used in this study is the same as in Ref. 7), so details of explanation are omitted. For the equation of motion and energy response of an SDOF structure excited by an earthquake motion, equations (2) and (3), normalized by the mass $m$ and the yield displacement $X_{y}$ of the structure, were also employed. These equations were integrated numerically by the linear acceleration method and the trapezoidal formula, respectively. SDOF structures were analyzed over the total duration $T_{t}$ (see Table 1) for each artificial earthquake and over $30 \mathrm{sec}$. from the beginning of all recorded earthquakes. In the numerical integration, equally spaced time intervals of $d t=0.02 \mathrm{sec}$. were adequately subdivided into smaller intervals, according to the values of both the damping factor $h$ and natural period $T^{7)}$.

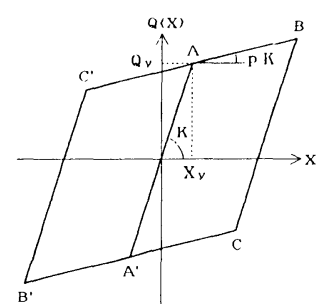

Fig. 2 Bi-linear model. 


$$
\begin{aligned}
& \ddot{\bar{X}}+2 h \omega \dot{\bar{X}}+\omega^{2} \bar{Q}(\bar{X})=-\ddot{\bar{X}}_{0} \\
& \int_{0}^{t} \stackrel{\ddot{X}}{\dot{X}} \cdot \dot{\bar{X}} d t+2 h \omega \int_{0}^{t} \dot{\bar{X}} \cdot \dot{\bar{X}} d t+\omega^{2} \int_{0}^{\bar{x}_{t}} \bar{Q}(\bar{X}) d \bar{X}=-\int_{0}^{t} \ddot{\bar{X}}_{0} \cdot \dot{\bar{X}} d t
\end{aligned}
$$

where, $\bar{X}, \dot{\bar{X}}$ and $\dot{\dot{X}}$ are the relative displacement, velocity and acceleration normalized by $X_{y}$, respectively. $h, \omega$ and $\bar{Q}(\bar{X})$ are the damping factor, natural circular frequency and normalized restoring force by $Q_{y}$, respectively, and $\dot{\bar{X}}_{0}$ is the acceleration of input earthquake motion normalized by $X_{y}$.

The third term on the left side in Eq. ( 3 ) is the hysteretic energy which is absorbed into the structure, and the right hand side indicates the input energy which applied to the structure by the earthquake motion. In addition, these are, as is obvious from Eq. (3), the energies normalized by the product of the mass $m$ and the square of the yield displacement $X_{y}^{2}$. Multiplying $X_{y}^{2}$ by these energies, the energies per unit mass will be shown in the next section, as the analytical results of the energy responses.

\section{ANALYTICAL RESULTS AND REMARKS}

In this study, for all combinations of the structural parameters and input earthquakes described in previous chapters, the numerical energy response analysis was carried out. Effective Fourier spectrum $F_{s e}$ for each input earthquake motion was also calculated. Then the authors examined the mutual relationship between the effective spectrum $F_{s e}$ and the response spectra $E_{s}$ of the elastic input energy $E_{i e}$ and hysteretic energy $E_{h \rho}$. In this paper, however, only the results for the structures which bear the damping factor $h=0.5 \%$, secondary slope $p=0.0$ and 0.5 and yield strength ratio $R=0.25$ and 0.5 are shown. Results both for these structures and for others show a quite similar tendency, although the value of the energy responses increases and decreases owing to the value of $h, R$ and $p$.

(1) Effective Fourier spectrum $\boldsymbol{F}_{\text {se }}$ for input earthquake motion

In this study, as was stated previously, attention is focused on the effective Fourier spectrum for the input earthquake motions. As can be seen from Eq. (4), this spectrum for any input earthquake is the one which modifies the corresponding ordinary Fourier spectrum with the transfer function of SDOF structures. So it is expected that this effective spectrum shows a closer relation to the energy responses of the structures than the ordinary Fourier spectrum which was concentrated on our previous study ${ }^{11)}$. Here, the Fourier amplitude of the effective spectrum $F_{s e k}$, corresponding to the structure with natural circular frequency $\omega_{k}\left(T_{k}\right)$ is calculated from the next equation.

$$
F_{\text {sek }}\left(h, \omega_{k}\right)=\int_{0}^{\infty} F(\omega) \cdot H\left(h, \omega_{k}, \omega\right) d \omega / \int_{0}^{\infty} H\left(h, \omega_{k}, \omega\right) d \omega
$$

in which $h$ and $\omega_{k}$ are the damping factor and natural circular frequency of a structure. $F(\omega)$ is the Fourier amplitude of an input earthquake motion for any circular frequency $\omega . H\left(h, \omega_{k}, \omega\right)$ is the transfer function for the acceleration response of an SDOF structure with $\omega_{k}$, as is defined by the following equation.

$$
H\left(h, \omega_{k}, \omega\right)=\sqrt{\left\{1+4 h^{2}\left(\omega / \omega_{k}\right)^{2}\right\} /\left[\left\{1-\left(\omega / \omega_{k}\right)^{2}\right\}^{2}+4 h^{2}\left(\omega / \omega_{k}\right)^{2}\right]}
$$

Figs. 3 (a) to (f) show the effective Fourier spectra $F_{s e}$ for all artificial earthquakes and recorded ones used in this study. These spectra are described by the use of each Fourier amplitude $F_{\text {sek }}$ which is related to each of the twenty different natural periods $T_{k}\left(\omega_{k}\right)$ of the structures with $5 \%$ damping, as was mentioned previously (section 3). From the comparison of Fig. 3 with Fig. 1, it'is found that the effective Fourier spectra $F_{s e}$, especially over short period range, are smoothed by the filtering effect of the transfer function, although the value of the spectra $F_{s e}$ for the artificial earthquakes depends on the value of the magnitude and epicentral distance $(M \cdot D)$ and ground condition GC in the same manner as that of ordinal Fourier spectra in Fig. 1.

(2) Energy response spectrum

Response spectra of the elastic input energy $E_{i e}$, obtained from elastic energy response analysis, are shown in Figs. 4 (a) to ( f ), against every natural period $T$ of the structures with $5 \%$ damping and every 


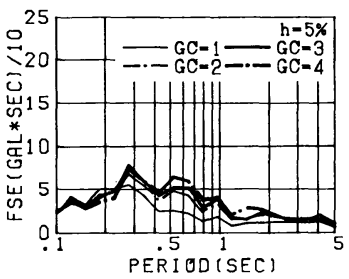

(a) $(M \cdot D)=A$

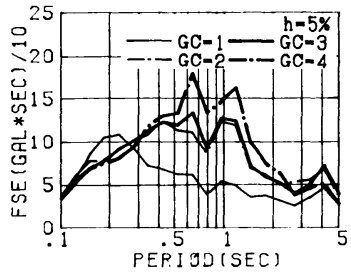

(c) $(M \cdot D)=C$

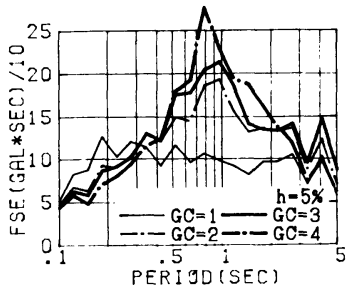

(e) $(M \cdot D)=E$

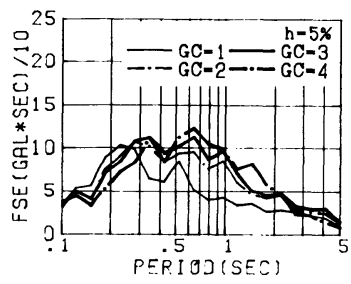

(b) $(M \cdot D)=B$

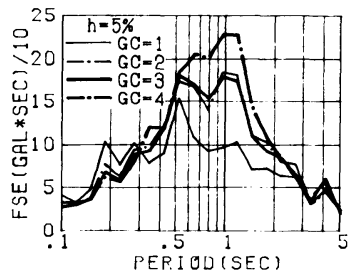

(d) $(M \cdot D)=D$

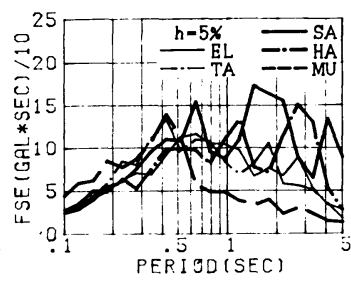

(f) Recorded

Fig. 3 Effective Fourier amplitude spectra for input earthquake motions.

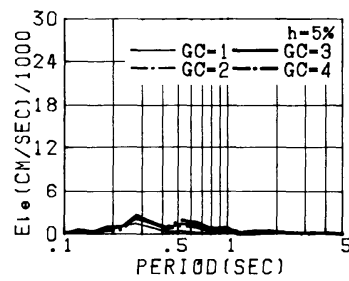

(a) $(M \cdot D)=A$

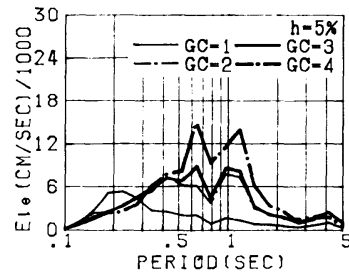

(c) $(M \cdot D)=C$

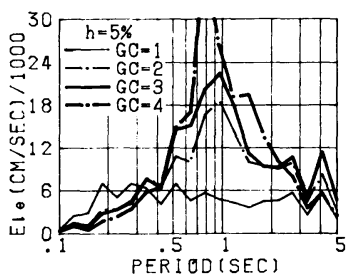

(e) $(M \cdot D)=E$

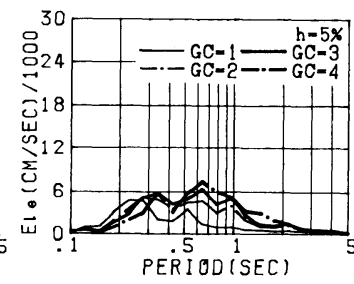

(b) $(M \cdot D)=B$

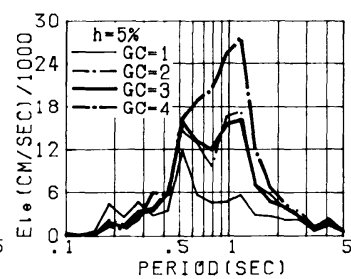

(d) $(M \cdot D)=D$

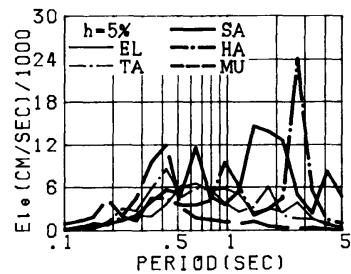

(f) Recorded
Fig. 4 Elastic input energy response spectra.

input earthquake motion given in this study. Comparing each corresponding figure in Fig. 4 and Fig. 3, a good correlation can be found between the periodical characteristics in the energy response spectra $E_{i e}$ (Fig. 4) and those in the effective Fourier spectra $F_{s e}$ (Fig.3), for each artificial and recorded earthquake. Also the large or small values of $E_{i e}$ at any natural period correspond well with the $F_{s e}$ results.

Against every $T$, the hysteretic energy response spectra $E_{h \rho}$ for each earthquake are illustrated in Figs. 5 (a) to (f). These are the results for the damping factor $h=5 \%$ and four pairs of yield strength ratios $R$ and secondary slopes $p$ as shown in the upper part of each figure. Comparing the results of $E_{h \rho}$ for $R=0.25$ and $R=0.5$, it can be seen that the effect of the yield strength ratio $R$ is significant, i. e., the value of $E_{h \rho}$ gets larger as $R$ becomes smaller. Also, the spectra for $R=0.25$ become smoother than those for $R=0.5$, because of the difference of elongation of the natural period $T$ followed by the degree of inelastic response of the structures. On the other hand, except for some parts of the short period range, this $E_{h p}$ value is scarcely affected by the secondary slope $p$. Next, as well as the elastic input energy $E_{i e}$ in Fig. 4, the value of the hysteretic energy $E_{h p}$ for artificial earthquakes becomes larger with the increasing number of ground conditions GC when the magnitude and epicentral distance $(M \cdot D)$ is the same as $(M \cdot D)$ $=[\mathrm{A}],[\mathrm{B}],[\mathrm{C}],[\mathrm{D}]$ or $[\mathrm{E}]$. This value also increases as the value of $(M \cdot D)$ gets larger when $\mathrm{GC}$ is the same as $\mathrm{GC}=1,2,3$ or 4 . Moreover, from Figs. 3 and 5 , it is noted that there is a good correlation between the spectra of this $E_{h \rho}$ and those of the effective Fourier spectra $F_{s e}$, as well as between $E_{i e}$ and $F_{s e}$.

(3) Spectrum for the ratio of square root of energy response to effective Fourier amplitude

It was found in the previous section that there is a close connection between the energy response spectrum and effective Fourier spectrum for each input earthquake motion. In order to make this point 


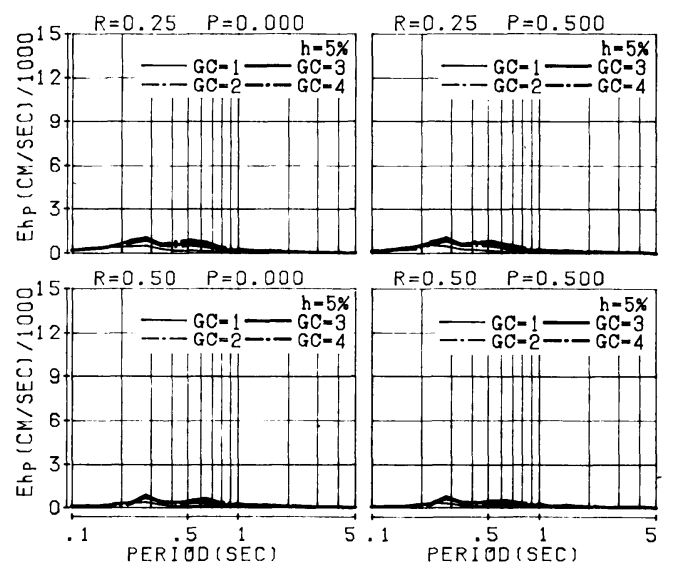

(a) $(M \cdot D)=A$
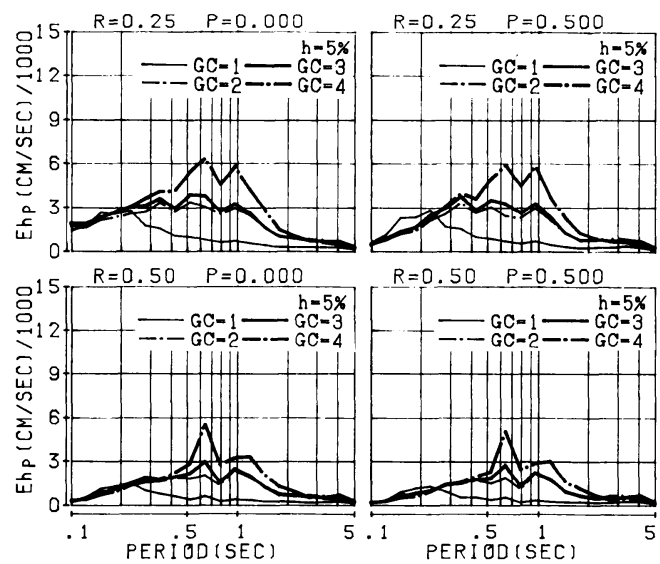

(c) $(M \cdot D)=C$
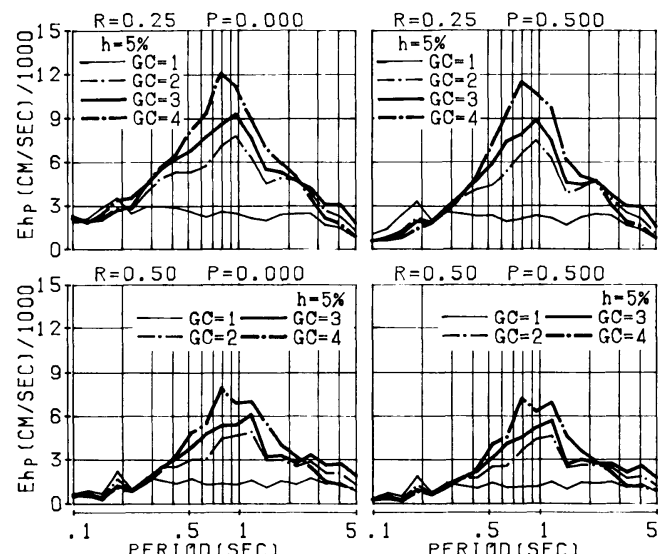

(e) $(M \cdot D)=E$

$R=0.25 \quad P=0.500$

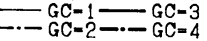
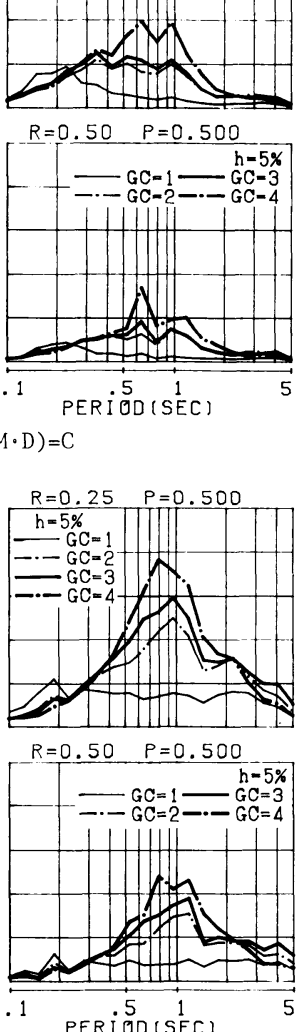
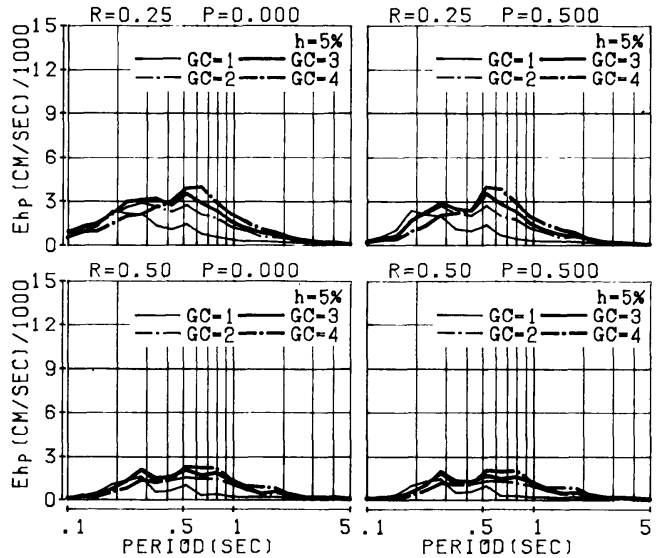

$R=0.50 \quad P=0.500$

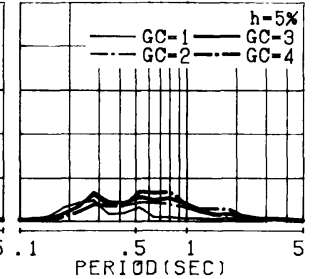

(b) $(M \cdot D)=B$

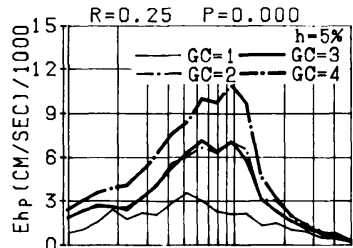

$R=0.25 \quad P=0.500$
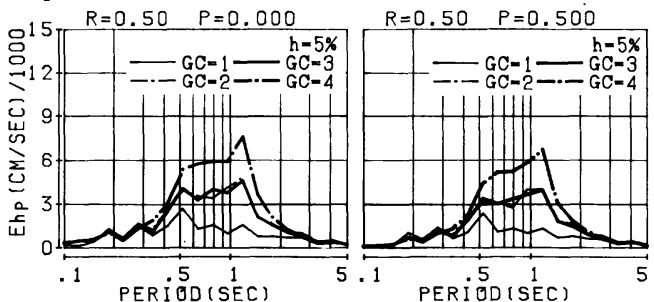

(d) $(M \cdot D)=D$
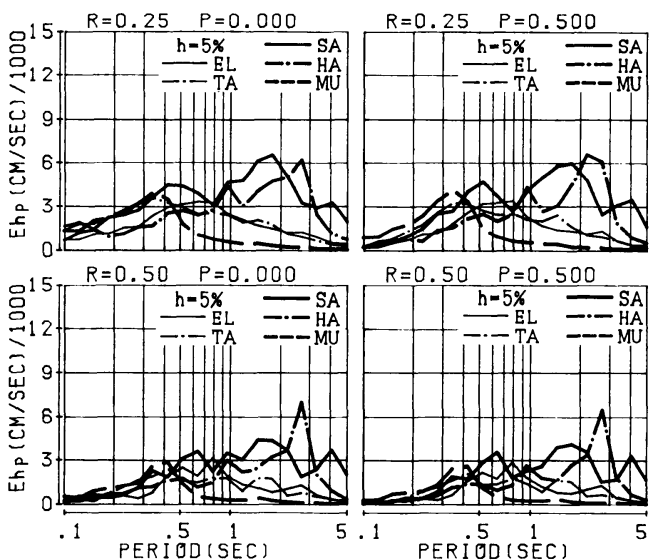

$R=0.50 \quad P=0.500$

Fig. 5 Hysteretic energy response spectra.

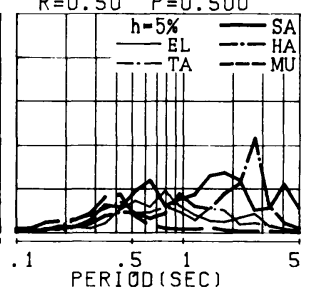

(f) Recorded

clearer, here, the ratio of the square root of the energy response $\sqrt{E_{i e k}}$ and $\sqrt{E_{h p k}}$ to the effective Fourier amplitude $F_{\text {sek }}$ for every natural period $T_{k}(k=1$ to 20$)$ is examined. The spectra for the results of this ratio $\sqrt{E_{i e k}} / F_{\text {sek }}$ and $\sqrt{E_{h \rho k}} / F_{\text {sek }}$ are shown in Fig. 6 and Fig. 7 , respectively. In each figure, the results for the twenty artificial earthquakes and five recorded ones are separated into figures (a) and (b). 


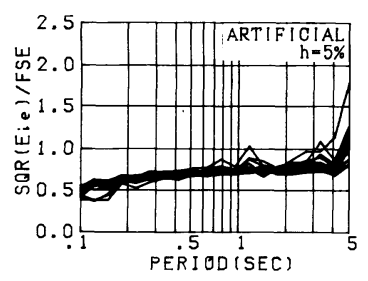

(a) Each value for all artificial earthquakes

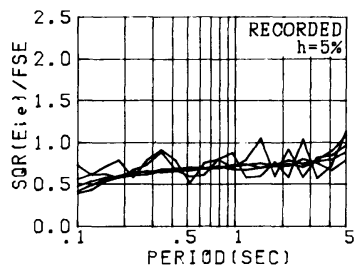

(b) Each value for all recorded earthquakes

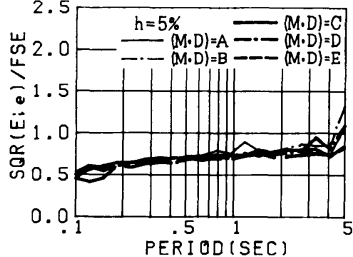

(c) Mean values for each group of artificial earthquake with same (iv. D)

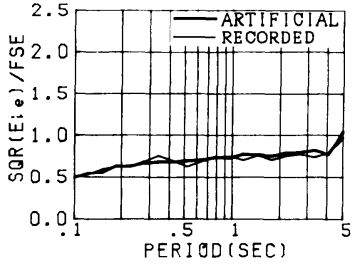

(d) Mean values for a11 recorded earthquakes and all artificial earthquakes

Fig. 6 Ratios of square root of elastic input energy to effective Fourier amplitude

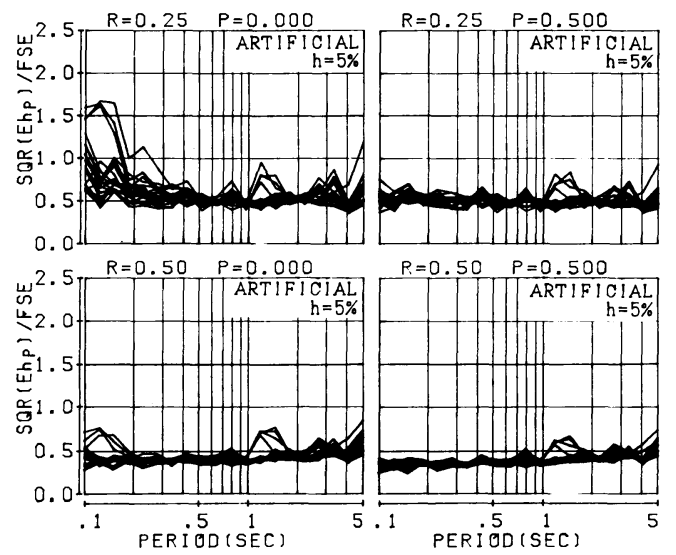

(a) Each value for all artificial earthquakes

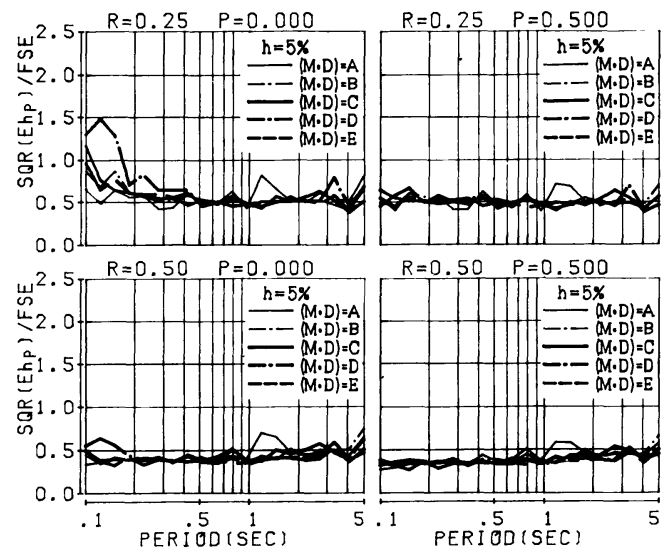

(c) Mean values for each group of artificial earthquakes with same $(M \cdot D)$
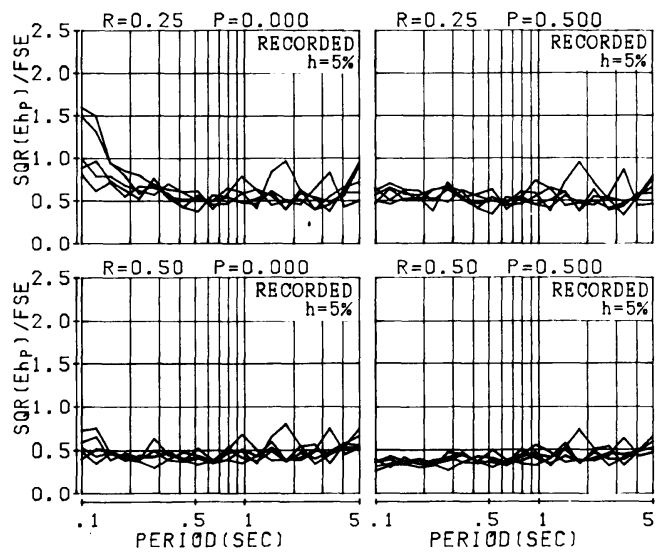

(b) Each value for a11 recorded earthquakes
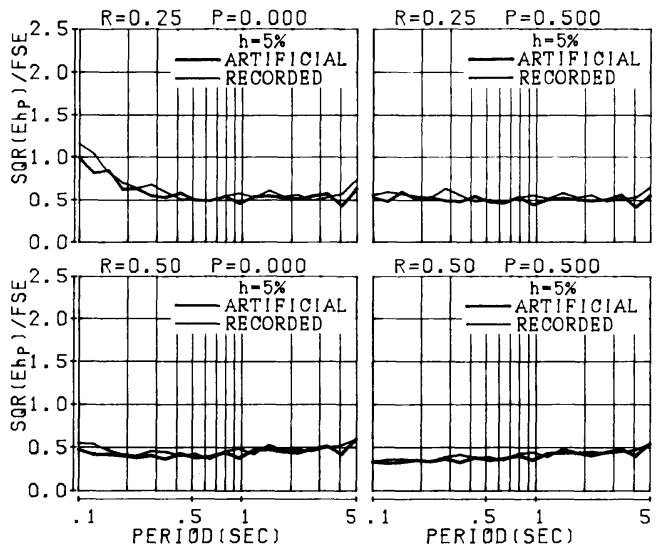

(d) Mean values for all recorded earthquakes and all artificial earthquakes

Fig. 7 Ratios of square root of hysteretic energy to effective Fourier amplitude.

Also, figure (c) shows the mean values for each group of four artificial earthquakes which vary in GC $(\mathrm{GC}=1,2,3$ and 4$)$ and bear the same $(M \cdot D)$ as $(M \cdot D)=[\mathrm{A}],[\mathrm{B}], \quad[\mathrm{C}]$, [D] and [E] . Furthermore, the comparison of the mean value for all artificial earthquakes with that for all recorded ones is given in figure $(\mathrm{d})$. As can be seen from Fig. 6 , the spectra for the ratio $\sqrt{E_{i e}} / F_{\text {se }}$ of each artificial earthquake coincide very well with one another, having no connection with the difference of $(M \cdot D)$ and GC values in the earthquakes (see figures (a ) and (c )). Comparing figure (b) with figure (a), it is found that the spectra for the recorded earthquakes in Fig. 6 ( b ) are almost all the same as the ones for the artificial earthquakes, although the scattering of the former is a little greater than that of the 
latter, and it is noted from figure ( $d$ ) that there is little discrepancy between these two mean spectra. This spectrum, however, depends a little on the natural period $T$ of the structures, i. e., the value of the spectrum gradually increases as the value of $T$ also increases.

Next, it is found from Fig. 7 that the scattering of the spectra for the ratio $\sqrt{E_{h p}} / F_{s e}$ amongst both the artificial earthquakes and recorded ones, as well as that for the ratio $\sqrt{E_{i e}} / F_{s e}$ mentioned above, is very limited excepting some ranges of the natural period $T$ for several earthquakes (figures (a), ( b ) and (c) ). Both the results for the artificial earthquakes and recorded ones are quite similar in the forms and values of their all and mean spectra, bearing no relation with the structural parameters $R$ and $p$ (figures (a), (b) and (d)). However, as is clear from figure (d), this ratio $\sqrt{E_{h p}} / F_{s e}$ depends slightly on the value of the structural parameters, i. e. , for the small value of the yield strength ratio $R$ and secondary slope $p$ as $R=0.25$ and $p=0.0$, this ratio in the short period range (less than $T=0.4$ sec.) increases as the period $T$ becomes smaller, owing to the elongation of the natural period $T$ followed with the intensive inelasticity of these structures. Also, in connection with the aforesaid dependence of $E_{h \rho}$ on the structural parameters, the value of the ratio $\sqrt{E_{h p}} / F_{s e}$ gets slightly larger with decreasing values of $R$ and $p$. The dependence of this $\sqrt{E_{h p}} / F_{s e}$ on $T$, however, is a little smaller than that of $\sqrt{E_{i e}} / F_{s e}$, i. e., in the case of $R=0.25$, this ratio takes nearly constant value for every natural period $T$. Even so the value for $R=0.5$ has a tendency to become larger as the period $T$ gets larger.

\section{(4) Regression equation}

As was just mentioned, the discrepancy amongst the results of the ratio $\sqrt{E_{i e}} / F_{s e}$ or $\sqrt{E_{h p}} / F_{s e}$ for every input earthquake motion is small enough to be able to be represented by their mean value. Therefore, if the regression equation for each mean value of these ratios is derived by relating to the structural parameters, it seems useful to estimate the value of $E_{i e}$ or $E_{h p}$ for a given structure directly from the effective Fourier spectrum for a selected earthquake, without the energy response analysis of the structure. From such a viewpoint, using the method of trial and error, the authors tried to derive the regression equations for each mean value of these ratios $\sqrt{E_{i e}} / F_{s e}$ and $\sqrt{E_{h p}} / F_{s e}$ for the twenty artificial earthquakes. The results obtained are as follows;

$$
\begin{aligned}
& \sqrt{E_{i e}} / F_{s e}=C_{0}+C_{1} T / 100+C_{2} /(100 \cdot T) \ldots \ldots \ldots \ldots \ldots \ldots \ldots \ldots \ldots \ldots \ldots \ldots \\
& \sqrt{E_{h p}} / F_{s e}=D_{0}+D_{1} /(100 \cdot T)+D_{2} T / 100+D_{3} p /(100 \cdot T)
\end{aligned}
$$

where $T$ and $p$ represent the natural period and secondary slope, and $C_{0}, C_{1}, C_{2}, D_{0}, D_{1}, D_{2}$ and $D_{3}$ are the coefficients given in Table 2, relating to the values of the damping factor $h$ and yield strength ratio $R$.

Figs. 8 and 9 show the regression curves for three different values of $h$, together with the mean values, so as to examine the accuracy of Eqs. (6) and (7) and the effect of the value of $h$ on the ratio $\sqrt{E_{i e}} / F_{s e}$ and $\sqrt{E_{h p}} / F_{s e}$. As is clear from these figures, the regression curves match the mean values well, and the ratio $\sqrt{E_{i e}} / F_{s e}$ is scarcely affected by the value of $h$. However the effect of $h$ value on the ratio $\sqrt{E_{h p}} / F_{s e}$ is not so small that it may be ignored, and this ratio becomes larger with the decrease of $h$.

Next, the ratio of $\sqrt{E_{i e}^{\prime}}$ and $\sqrt{E_{h p}^{\prime}}$ estimated by Eqs. (6) and (7) to $\sqrt{E_{i e}}$ and $\sqrt{E_{h p}}$ obtained from energy response analysis, are illus-

\begin{tabular}{|c|c|c|c|c|c|c|c|c|}
\hline \multirow{2}{*}{ h $(\%)$} & \multicolumn{3}{|c|}{ SQR (Eie)/Fse } & \multicolumn{5}{|c|}{$\mathrm{SQR}(\mathrm{EhP}) / \mathrm{Fse}$} \\
\hline & $\mathrm{CO}$ & C 1 & C 2 & $\mathrm{R}$ & D O & D 1 & D 2 & D 3 \\
\hline \multirow{3}{*}{2.5} & \multirow{3}{*}{0.716} & \multirow{3}{*}{ 2. 746} & \multirow{3}{*}{-1.694} & 0.25 & 0.526 & 1.622 & 5.001 & -6.988 \\
\hline & & & & 0.50 & 0.457 & 2.819 & 0.369 & -1.931 \\
\hline & & & & 0.75 & 0.335 & 2.755 & -0.815 & -1.354 \\
\hline \multirow{3}{*}{5.0} & \multirow{3}{*}{0.718} & \multirow{3}{*}{ 4. 010} & \multirow{3}{*}{-2.175} & 0.25 & 0.452 & 1.861 & 4.656 & -7.155 \\
\hline & & & & 0.50 & 0.371 & 3.082 & 0.699 & -2.470 \\
\hline & & & & 0.75 & 0.257 & 2. 988 & -0.560 & -1.270 \\
\hline \multirow{3}{*}{7.5} & \multirow{3}{*}{0.723} & \multirow{3}{*}{4.879} & \multirow{3}{*}{-2.611} & 0.25 & 0.042 & 1.619 & 3.993 & -6.733 \\
\hline & & & & 0.50 & 0.336 & 2.750 & 0.717 & -2.650 \\
\hline & & & & 0.75 & 0.222 & 2.780 & -0.472 & -1.250 \\
\hline
\end{tabular}

Table 2 Coefficients of regression Eqs. (6) and (7).

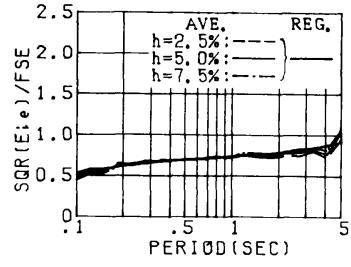

Fig. 8 Mean values and regression curve for $\sqrt{E_{i e}} / F_{\text {se }}$.

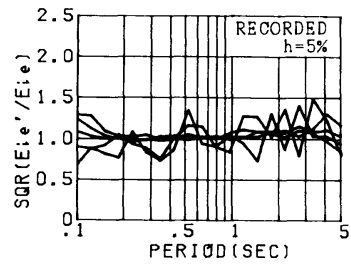

Fig. $10 \sqrt{E_{i e}^{\prime} / E_{i e}}$ for recorded earthquakes. 

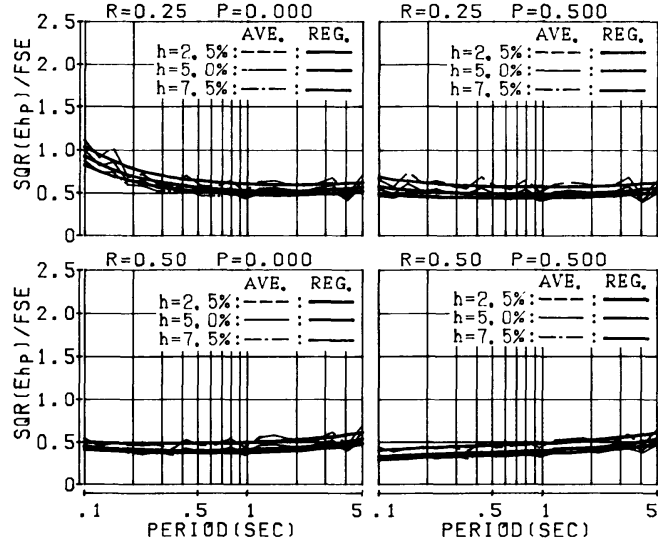

Fig. 9 Mean values and regression curves for $\sqrt{E_{h \rho}} / F_{\text {se }}$.
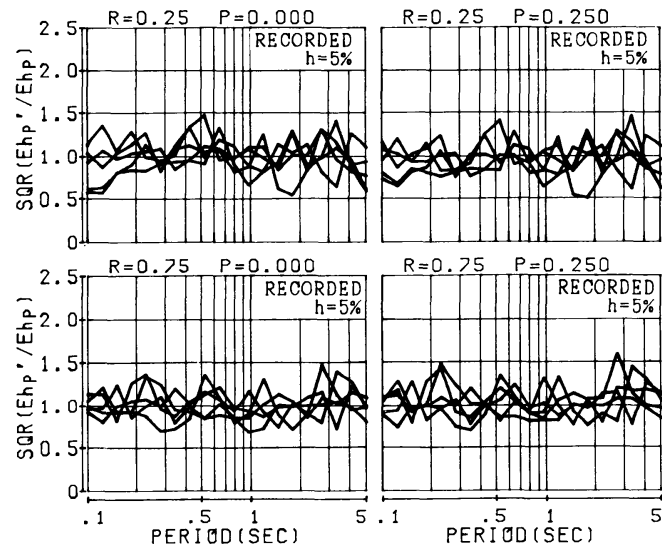

Fig. $11 \sqrt{E_{h \rho}^{\prime} / E_{h \rho}}$ for recorded earthquakes.

trated in Figs. 10 and 11, respectively. Here these are the results for the structures with damping factor $h=5 \%$ and for the five recorded earthquake motions selected in this study. It can be seen from these figures that all values of this ratio for every earthquake remain within the range of 0.5 to 1.5. So it is said that the accuracy of the estimate $\sqrt{E_{i e}^{\prime}}$ and $\sqrt{E_{h p}^{\prime}}$ is relatively precise.

Consequently, following this study, it would seem to be possible to estimate the value of the elastic input energy $E_{i e}$ and hysteretic energy $E_{h p}$ of any structure directly from it's structural parameters and the effective Fourier spectrum for each input earthquake motion, to a fair degree of accuracy, providing we can derive a more practical and reliable regression equation for the ratio of $\sqrt{E_{i e}} / F_{s e}$ and $\sqrt{E_{h \rho}} / F_{s e}$.

\section{CONCLUSIONS}

This is a fundamental study to find out a practical way to estimate the value of the elastic input energy $E_{i e}$ and hysteretic energy $E_{h p}$ of structures subjected to strong earthquake motions. In this study, twenty artificial earthquakes, each varying in frequency characteristics and duration, were generated. The numerical energy response analysis for wide ranging bi-linear SDOF structures was carried out by the use of these artificial earthquakes and five recorded ones. Then, based on the analytical results, the mutual relation between the effective Fourier spectra $F_{s e}$ for the input earthquakes and response spectra of $E_{i e}$ and $E_{h \rho}$ was examined. Finally the regression equations for $\sqrt{E_{i e}} / F_{s e}$ and $\sqrt{E_{h \rho}} / \dot{F}_{s e}$ were derived, and the accuracy of these equations was also investigated.

The results obtained in this study are summarized as follows;

(1) There is a good correlation between the response spectrum for the elastic input energy $E_{i e}$ and hysteretic energy $E_{h p}$ of the structures and the effective Fourier spectrum $F_{s e}$ for the input earthquake motion, i. e., the large and small values of $E_{i e}$ and $E_{h \rho}$ at any natural period of the structures correspond well to the $F_{\text {se }}$ results.

(2) In connection with the result (1), the value of $\sqrt{E_{i e k}} / F_{\text {sek }}$ and $\sqrt{E_{h p k}} / F_{\text {sek }}$, which is the ratio of the square root of the energy response $\sqrt{E_{i e k}}$ and $\sqrt{E_{h \rho k}}$ for the structure with any natural period $T_{k}$ to the corresponding effective Fourier amplitude $F_{\text {sek }}$, is scarcely affected by the difference of the input earthquake motions, i.e., the scattering of the spectra for these ratio amongst the input earthquake motions is small enough for them to be represented by their mean value. Also there is a good functional relation between the spectrum for each mean value of these ratios and structural parameters, such as natural period $T$, damping factor $h$, yield strength ratio $R$ and secondary slope $p$. From these facts, it is possible to derive the regression equations for these mean values, relating to the structural parameters.

(3) When the values of the structural parameters $T, h, R$ and $p$ of a structure and the effective 
Fourier amplitude spectrum $F_{s e}$ for the input earthquake motion are given, the value of the elastic input energy $E_{i e}$ and hysteretic energy $E_{h \rho}$ of the structure is estimated using regression Eq. (6) and Eq. ( 7 ), respectively, with relative accuracy.

These results are obtained mainly through the limited numerical analysis for twenty artificial earthquakes, whose acceleration response factor spectra agree with the target ones shown in the Japanese specifications for road bridges $\mathrm{V}$. So in order to put the results in this study to practical use, it seems to be necessary to examine and to verify the corresponding relation between the results of the artificial earthquakes used in this study and the results of many other actual/recorded ones, and this subject is worthy of more discussion in the future.

\section{ACKNOWLEDGMENTS}

Some of the recorded earthquakes used in this study are records which were originally reported ${ }^{15)}$ by the port and harbour research institute, ministry of transport, Japan, and were collected by Goto et al. ${ }^{16)}$. So the authors would like to express their appreciation to the persons concerned with this. The authors also wish to thank Dr. Tsutomu Sawada, associate professor of Tokushima University, for his kind cooperation in generating the artificial earthquakes.

\section{REFERENCES}

1) Housner, G. W. : Behavior of Structures During Earthquakes, Proc. of ASCE, Vol. 85, No. EM 4, pp. 109-129, Oct., 1959.

2) Kato, B. and Akiyama, H. : Energy Input and Damages in Structures subjected to severe Earthquakes, Proc. of AIJ, No. 235, pp. 9-18, Sept., 1975 (in Japanese).

3) Ohno, T., Nishioka, T. and Fujino, Y. : Quantitative Estimation of Plastic Energy absorbed in Structures subjected to Seismic Excitation, Proc. of JSCE, No. 333, pp.91-99, May, 1983 (in Japanese).

4) Yamada, Y., Iemura, H. and Nishimura, I. : Evaluation of Aseismic Resistance of R. C. Bridge Pier Models based on Hysteretic Energy, Proc. of the 17 th JSCE Conference on Earthquake Engineering, pp.231-234, July, 1983 (in Japanese).

5) Fajfar, P. and Fischinger, M. : Parametric Study of Inelastic Response to Some Earthquakes Recorded in Southern Europe, Proc. of 8 th WCEE, pp.75-82, 1984.

6) Hirao, K., Sawada, T. and Nariyuki, Y. : On the Hysteretic Energy Absorption in Structures under Strong Earthquake Motion, Proc. of EASEC-1, Vol.3, pp.1904-1915, Jan., 1986.

7) Hirao, K., Sawada, T., Nariyuki, Y. and Sasada, S. : On the Relation between Hysteretic Energy and Elastic Responses of Single Degree of Freedom Systems under Strong Earthquake Motions, Proc. of JSCE, No. 368/I-5, pp. 401-410, Apr., 1986 (in Japanese).

8) Zahrah, T.F. and Hall, W. J. : Earthquake Energy Absorption in SDOF Structures, Proc. of ASCE, Vol. 110, No. ST 8 , pp. 1757-1772, Aug., 1984.

9) Matsushima, M. and Matsui, K. : The Effect of Ground Motion upon Simple Structures, Proc. of Structural Engineering, Vol. 32 A, pp. 815-824, Mar., 1986 (in Japanese).

10) Zhu, T. J., Tso, W. K. and Heidebrecht, A. C. : Effect of Peak Ground a/v Ratio on Structural Damage, J. Struct. Engrg. , ASCE, Vol. 114, No. 5, pp. 1019-1037, May, 1988.

11) Hirao, K., Sawada, T., Nariyuki, Y. and Sasada, S. : The Effect of Frequency Characteristics and Duration of Input Earthquake Motion on the Energy Response of Structures, Proc. of JSCE, No.386/I-8, Structural Eng. /Earthquake Eng. Vol. 4, No. 2, pp. 381 s-390 s, Oct., 1987.

12) Japanese Road Association: Specifications for Road Bridges V, pp.111-125, May, 1980 (in Japanese).

13) Sawada, T. and Hirao, K. : A Generation Method of Artificial Earthquake complying with Target Response Spectrum, Proc. of the 17 th JSCE Conference on Earthquake Engineering, pp.49-52, July, 1983 (in Japanese).

14) Sawada, T., Nagae, M. and Hirao, K. : A Definition for Duration of Earthquake Ground Motion by Phase Differences and its Statistical Analysis, Proc. of JSCE, No.368/I-5, pp.373-382, Apr., 1986 (in Japanese).

15) The Port and Harbour Research Institute of Ministry of Transport, Japan : Annual Report on Strong-Motion Earthquake Records in Japanese Ports, Technical Note of the Port and Harbour Research Institute, No. 98, Mar., 1970 (in Japanese).

16) Goto, H., Kameda, H., Sugito, M. and Imanishi, N. : Correction of SMAC-B 2 Accelerograph Records by Digital Filter, Proc. of JSCE, No. 277, pp. 57-69, Sept., 1978 (in Japanese). 